\section{Erster Vorsitzender}

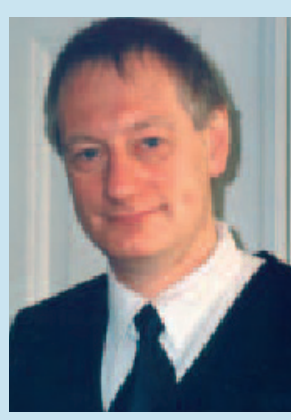

Prof. Dr. Albrecht

Bufe

Experimentelle

Pneumologie,

Universitäts-

klinik Berg-

mannsheil,

Bochum

Stellvertretender Vorsitzender

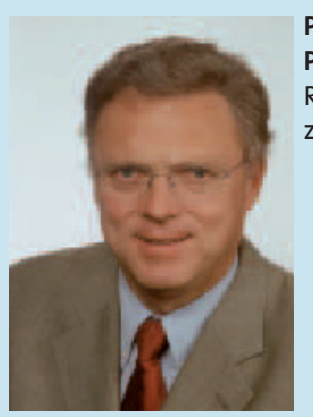

Prof. Dr. Carl

Peter Bauer

Rehabilitations-

zentrum Gaißach

\section{Schatzmeister}

\section{Prof. Dr. Jürgen Seidenberg}

Zentrum für Kinder- und Jugendmedizin,

Elisabeth-Kinderkrankenhaus, Oldenburg

\section{Schriftführer}

Dr. Frank Friedrichs

Kinderarztpraxis Laurensberg, Aachen

\section{Weitere Vorstandsmitglieder}

— Prof. Dr. Johannes Forster, Freiburg

_ Priv.-Doz. Dr. Günter Frey, Aue

_ Priv.-Doz. Dr. Matthias Kopp, Freiburg

— Dr. Wolfgang Lässig, Halle

— Dr. Ernst Rietschel, Köln

\section{Kooptierte Vorstandsmitglieder}

— Dr. Frank Ahrens, Hamburg

— Dr. Peter Eberle, Kassel

— Dr. Philippe Eigenmann, Genf, Schweiz

— Dr. Isidor Huttegger, Salzburg, Österreich

— Dr. Thomas Lob-Corzilius, Osnabrück

_ Prof. Dr. Bodo Niggemann, Berlin

— Dr. Rüdiger Szczepanski, Osnabrück

\section{Geschäftsstelle}

Ute Lohschelder-Dreuw

GPA-Geschäftsstelle, Rathausstraße 10,

52072 Aachen

Tel.: (02 41) 98 oo-4 86

Fax: (02 41) 98 o0-2 59

E-Mail:gpa.ev@t-online.de

\title{
Wechsel im Vorstand der GPA
}

$\mathrm{D}$ ie Gesellschaft für Pädiatrische Allergologie und Umweltmedizin e.V. (GPA) hat im Rahmen ihrer Mitgliederversammlung am 25. Januar 2008 in Höhenried einen neuen Vorstand bestimmt. Dabei gab es einen Wechsel an der Spitze: Neuer erster Vorsitzender ist Prof. Dr. Albrecht Bufe. Er löst Prof. Dr. Carl Peter Bauer ab, der jetzt als stellvertretender Vorsitzender fungiert. Schatzmeister bleibt unverändert Prof. Dr. Jürgen Seidenberg, Dr. Frank Friedrichs fungiert weiter als Schriftführer. Der neue Vorstand ist für die Dauer von drei Jahren gewählt. Seine komplette Zusammensetzung ist in der Randspalte links wiedergegeben.

Außerdem wurde die Gründung von sechs neuen Arbeitsgruppen beschlossen: 1. Anaphylaxie (Sprecher Dr. Ernst Rietschel)
2. Atopisches Ekzem (Sprecher Dr. Frank Ahrens)

3. Nahrungsmittelallergie (Sprecher Prof. Dr. Bodo Niggemann)

4. Prävention (Sprecher Priv.-Doz. Dr. Matthias Kopp)

5. Spezifische Immuntherapie (Sprecher Dr. Peter Eberle)

6. Umweltmedizin (Sprecher Dr. Thomas Lob-Corzilius)

Die Sprecher der Arbeitsgruppen wurden komplett neu in den Kreis der kooptierten Vorstandsmitglieder aufgenommen. Als weitere kooptierte Vorstandsmitglieder wurden in ihrer Funktion als Sprecher der pädiatrischen Allergologie in Österreich und in der Schweiz wieder Dr. Isidor Huttegger, Salzburg, und Dr. Philippe Eigenmann, Genf, benannt.

\section{GPA-Förderpreis 2008 fortgesetzt}

$\mathrm{D}$ ie Gesellschaft für Pädiatrische Allergologie und Umweltmedizin e.V. (GPA) vergibt 2008 zum sechsten Mal in Folge den Förderpreis Pädiatrische Allergologie, der vom Diagnostikaunternehmen Phadia gestiftet wird. Der Preis besteht aus einer finanziellen Zuwendung in Höhe von $5.000 €$, die der weiteren finanziellen Sicherung des gewürdigten Projekts dienen soll. Mit dem Förderpreis sollen Einzelpersonen oder Gruppen ausgezeichnet werden, die sich in herausragender Weise um die Verbesserung der Situation allergiekranker Kinder verdient gemacht haben. Besonders förderwürdig sind Projekte, die der Umsetzung wissenschaftlicher Erkenntnisse in die Praxis dienen. Dies kann sowohl durch wissenschaftliche Aktivitäten, Publikationen in der Fachoder Laienpresse, Öffentlichkeitsaktionen oder andere Aktivitäten geschehen.

Bisher ausgezeichnet mit dem Förderpreis Pädiatrische Allergologie wurden

— 2007 Prof. Dr. Erika von Mutius,

— 2006 die Arbeitsgemeinschaft Asthmaschulung im Kindes- und Jugendalter (AGAS),
— 2005 der Deutsche Allergie- und Asthmabund e.V. (DAAB),

— 2004 der saarländische Qualitätszirkel „Allergologie, Pneumologie und Asthmaschulung im Kindesalter" und

— 2003 die gemeinnützige Kinderumwelt $\mathrm{GmbH}$ in Osnabrück.

Die Verleihung des Preises findet anlässlich der Jahrestagung der Gesellschaft für Pädiatrische Allergologie und Umweltmedizin im September 2008 in Erfurt statt.

Impressum „GPA-Nachrichten“"

Verantwortlich für den Inhalt

Dr. Ernst Rietschel, Köln

Redaktion

Markus Seidl

Verlag

(c) Urban \& Vogel GmbH, München

GPA-Geschäftsstelle

Ute Lohschelder-Dreuw, Aachen

Tel.: (02 41) 98 oo-4 86

Fax: (02 41) 98 o0-2 59

E-Mail: gpa.ev@t-online.de 